\title{
53 CORE PRINCIPLES AND GUIDANCE IN RELATION TO CPR STATUS
}

10.1136/bmjspcare-2011-000053.53

R George', E Sherwen'2, C Regnard ${ }^{3}{ }^{1}$ Guys and St Thomas's NHS Foundation Trust, London, UK; ${ }^{2}$ NHS National End of Life Care Programme, London, UK; ${ }^{3}$ St Oswalds Hospice, Newcastle upon Tyne, UK

The End of Life Care Strategy (DH 2008) highlights the importance, within Care Planning, of clear and appropriate discussion and decisions about CPR. These decisions may change over time with clinical deterioration and/or therapeutic reversibility. One point at which this becomes critical is when a patient moves between care settings for example, with some clinical crisis or when care is delivered better elsewhere.

Currently, while organisations have crafted their own policies, small but critical variations exist, together with the assumption that 'our policy ends at our front door'. This has profound implications for transport and receiving services because this hiatus, for anyone in transit who does not want CPR, will inevitably make them vulnerable to inappropriate intervention. This can be overcome by harmonising CPR policies between partner organisations or across localities to ensure that there are clear ways in which people are managed when being moved.

There are excellent examples of work nationally including the Unified Policy being implemented across South Central SHA, DNACPR Principles from South East Coast SHA, and the unified policy being developed within the East of England SHA.

The National End of Life Care programme has scoped current work with key partners to produce a set of Core Principles and guidance for managing the intersection of policies where unification has not or cannot yet be achieved. This is aimed at organisations wishing to work together to ensure that policies connect to achieve smooth transition for the individual that safeguards choice, reduces unwanted and unnecessary attempts at resuscitation and promotes a dignified death. 